\title{
Study and Analysis of Image Segmentation Techniques for Food Images
}

\author{
Shital V. Chavan \\ Department of Computer Engineering \\ Pimpri Chinchwad College of Engineering \\ Pune-44
}

\author{
S. S. Sambare \\ Department of Computer Engineering \\ Pimpri Chinchwad College of Engineering \\ Pune-44
}

\begin{abstract}
As a person across the world is now becoming more caring about their health and weight. Many systems are available to measure the calorie intake from the food images before and after eating. Accuracy of the calorie measuring system is depending on food image analysis. The proper analysis of an image is based on image segmentation technique and is one of the important steps in image analysis. Multiple image segmentation techniques exist for extracting requires objects from an image. In this paper different image segmentation techniques based on Edge Detection, Morphological Operation, Threshold and Clustering based techniques studied and analyzed for segmenting different food images. Different image segmentation techniques implemented in MATLAB and then analyzed.
\end{abstract}

\section{Keywords}

Image Segmentation, Thresholding, Edge Detection, Morphological Operations, Clustering.

\section{INTRODUCTION}

Normally food images are used in calorie measurement systems to calculate how much calorie user eaten, by analyzing food image before and after eating. From normal human eye vision nobody accurately say how much calorie is getting from food. So there is an urgent need of control and monitoring system required to control on dietary intake. Computer vision technology, which helps in processing the any type of images and extracting meaningful information from images. Images are considered as one of the most important medium to carry information about content of images which are present in images [1]. In image analysis, image segmentation is one of the most important step for processing images and good segmentation results into accurate results. Image segmentation is the process of dividing the digital image into multiple segments, i.e. set of pixel and pixels in a region are similar according to some similarity criteria such as color, intensity or texture, to identify objects and boundaries present in an image [2].Image segmentation techniques are mainly dividing into three categories as shown in figure 1.

Thresholding methods convert color image into gray level image and separate out area of interest from background. In thresholding 1 represent foreground image and 0 represent background image. In Edge Detection method edges are extracted and different regions are separate out. In clustering method basically k-means clustering is used and which cluster the pixels which are similar to each other into same cluster. Clustering is unsupervised technique. In region based segmentation image is partitions into different regions based on predefined condition or seed point.

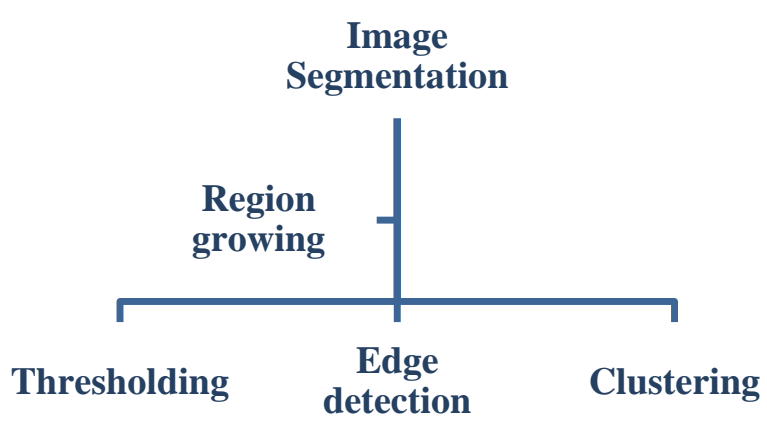

Figure 1: Image Segmentation Techniques

\section{RELATED WORK}

Parisa Pouladzadeh, Shervin Shirmohammadi and Rana AlMaghreb et al [3]. In this paper, proposed a food calorie and nutrition measurement system that can help dietitians and patients to measure and manage their daily food intake. The system uses nutritional fact tables and food images. System uses the built-in camera of such mobile devices and records a photo of the food before and after eating to measure the how much of calorie and nutrient components user have eaten. Edge detection method is used to separate out different regions present in image and after that SVM classification technique is used to classify extracted regions. From that calorie measurement is done, but edge detection method not work well for image which having fruit salad or food portions which are mixed.

Nandhini. P, Dr. J. Jaya et at [4]. This paper uses various image processing steps to provides an efficient method for identifying the defected parts in food materials. Steps in image processing are image acquisition, Preprocessing of image, image segmentation, feature extraction and classification. In proposed framework they compare different types of filters and after comparison hybrid median filter was selected as the filter with the high PSNR value and is used in preprocessing. Two image segmentation techniques such as Colour based binary Image segmentation, Particle swarm optimization are compared and finally got that for food quality evaluation colour based binary image segmentation is well preformed.

Hamirul Aini Hambali, Sharifah Lailee Syed Abdullah, Nursuriati Jamil and Hazaruddin Harun et al [5]. This paper proposed a rule-based segmentation method to segment natural images correctly and accurately. To segment the images of interested object this method uses IF-THEN algorithm. Firstly input colored image in converted into RGB colour format and then Identify colour of investigated object 
if colour is black then apply adaptive thresholding otherwise apply adaptive k-means clustering method. Adaptive thresholding is work well for image with black colour objects. Otsu, K-means and Fuzzy C-means and proposed method, all four segmentation methods are implemented on fruit images and based on visual and quantitative evaluations their performances are compared.

Marios Anthimopoulos, Joachim Dehais, Peter Diem and Stavroula Mougiakakou et al [6]. In this paper, they proposed novel methodology for the segmentation and recognition of multi-food images. The proposed method is implemented for type 1 diabetic patients for carbohydrate counting. Firstly the plate is segmented using pyramidal mean-shift filtering and a region growing algorithm. Then support vector machine classification technique is used to classify segmented regions into one of six different major food classes. The proposed food segmentation algorithm is based on color information and consists of CIELAB conversion, pyramidal mean-shift filtering, region growing, region merging, and plate detection or background subtraction and finally food recognition.

Y. He, N. Khanna, C.J. Boushey and E.J. Delp et al [1]. This paper presented a quantitative evaluation of automatic image segmentation methods for food image analysis used for dietary assessment. Dietary assessment is the process of determining what and how much eats during the course of a day and it is necessary for understanding the relationship between diet and health. In this paper three segmentation methods are examined active contours, normalized cuts, and local variation. In active contours the segmentation result is highly dependent on the initial contours and relative position of food items. Normalized Cuts is a graph based image segmentation method which treats the segmentation problem as the partition of a graph. Each image pixel is assigned as a node of a graph. The weight of an edge between image pixels is a measure on their intensity similarity and spatial proximity. The entire image is treated as a weighted and undirected graph. Similar to normalized cuts, local variation is also a graph based image segmentation method. Weights on each edge measure the dissimilarity between pixels. Inter distance between any two region is high. An experimental result shows that local variation is more stable to the changes of input parameters than the other two segmentation methods. Complexity of active contours increases as the number of initial contours increases.

Domingo Mery, Franco Pedreschi et al [7]. In this paper, a robust algorithm for segmenting food image from a background is proposed using colour images. The proposed method is implemented into three steps: (i) compute high contrast monochrome image of colour input image (ii) then apply global thresholding and (iii) morphological operations are apply in order to fill the holes which are presented in the segmented binary image. The suggested global thresholding clearly separate out are of interest from background. But this proposed method is not suitable for image having mix food; it shows output like single segmented image.

Table 1 shows comparative analysis of all papers.

\section{IMAGE SEGMENTATION METHODS}

Image Segmentation is the process of dividing colour or gray level images into different regions or segments as per the area of interest. Segmentation is used for object recognition, boundary estimation, image compression, image editing [4]. The main objective of image segmentation is to divide the image for further image analysis and arrange pixels into high level representation for further analysis. Multiple segmentation algorithms are exits, choice of particular segmentation technique is depend upon for which application it is useful and type of image which is being processed for extracting information from the image.

\subsection{Segmentation based on Thresholding}

Image segmentation based on thresholding is quite simple than other segmentation methods, here single value is selected as threshold and used to segment the image mostly into foreground and background segments. Value taken as thresholding is $\mathrm{T}$ and image is $\mathrm{I}$, for any pixel $\mathrm{I}(\mathrm{x}, \mathrm{y})$ intensity value greater than $T$ replace that pixel intensity value by 1 otherwise replace it by 0 . There are two methods namely global thresholding and local thresholding [2]. When there is intensity distribution between the foreground pixel intensity value and background pixel intensity value which are very distinct from each other then global thresholding method is used. Otsu method is one of the common methods of global thresholding. In Multilevel thresholding by using Otsu method multiple threshold values are used to segment the input image.

\subsection{Segmentation based on Edge Detection}

Edge detection method is used to solve image segmentation by detecting the edges or pixels between different regions that have sudden transition in intensity values are extracted and linked to form closed object boundaries. The result is in a binary image. Edge detection having three steps namely Filtering, Enhancement and Detection [8]. Most commonly used edge detection methods are Canny, Sobel, Prewitt, Robert these all are first derivative operators. When there is sudden change in intensity value near edge and there is little noise in image then gradient based method works well. This method involves roll together gradient operators with the image. High value of the gradient magnitude is possible place of rapid transition between two different regions. These are edge pixels, they have to be linked to form closed boundaries of the regions. Common edge detection operators used in gradient based method are Sobel operator, canny operator, Laplace operator, Laplacian of Gaussian (LOG) operator, canny is most promising one, but takes more time as compared to Sobel operator [2]. Morphological operations are also used to extract the shape of images. It is mostly apply on binary images Dilation and erosion are one of the morphological operations. In dilation operation edges of objects in binary image are becoming broad or thick. In erosion operation edges of objects in binary image shrinks or thins.

\subsection{Region based Segmentation}

Segmentation method based on region is relatively easy as compare to edge detection method and more effective to noise. In edge detection method image is segmented based on changes in intensity near edges at great rate and in region based methods, image is partition into regions that are similar according to a set of predefined condition or seed point [2]. Region based segmentation include two methods Region growing, region Splitting and Merging.

\subsubsection{Region Growing}

In region growing method, pixels are grouped into different region based on predefined condition or initial seed point. In region growing method first select a seed particle from input image, and then Select set of the criteria to find out similar seed based on color or intensity value and after that set stopping rule. Region is growing as per the similar properties 
between the seed pixel and neighboring pixels. When no more pixels that matches the predefined criteria region growing stops.

\subsubsection{Region Splitting and Merging}

Instead of choosing seed points, divide an image into a set of unconnected regions and then merge the regions to satisfy the conditions of image segmentation [2]. First image is split into small regions till regions with mandatory degree of homogeneity are formed. The splitting of the phase has an impact on the overall segmentation of the image. This results in over segmentation of the image. And then merging step is followed. Disadvantage of region splitting and merging techniques is complex and take a lot of time.

\subsection{Segmentation based on Clustering}

There are two types of learning task unsupervised learning task and supervised learning task, clustering is unsupervised task, there is no need of training set required to cluster the input data here data is pixels of input images[9]. Clustering is done on the basis of similarity criteria defined between pixels. The pixels are grouped into clusters in such manner that intra cluster similarity is maximum and inter cluster similarity is minimum. K-means clustering and fuzzy clustering these two methods are basically used to cluster the input image [2].

\subsubsection{Hard Clustering}

$\mathrm{K}$-means clustering is used as hard clustering. In hard clustering sharp boundaries are assumed between clusters. Same pixel is not found in two clusters at a time [2]. In kmeans clustering initial cluster center are randomly choose and make sure that these cluster centers are far away from each other and then by using any distance measuring method calculate distance between each pixel value with each cluster center and assign that pixel to that cluster having less distance between pixel value and cluster center. Once whole image is scanned then repeat same procedure and recalculate cluster center values by calculating mean value of all pixel values present in that cluster. Until no more change in cluster center values this procedure is repeated. Finally image is getting clustered into different clusters [9].

\section{EXPERIMENTAL RESULTS AND DISCUSSIONS}

To study different image segmentation technique, food input images are taken from web. Basically input images having different fruits and vegetables, all input images having different background and size. For experiment the image size taken as $200 * 200$ and 10 different images are taken as input. Figure 2 and Figure 3 shows experimental result of image segmentation. Image segmentation techniques based on thresholding, edge detection and clustering are applied on input images to segment the input image. Segmentation results are discussed on the basis of visual evaluations and quantitative. In thresholding technique global thresholding and multilevel colour thresholding is applied on input images. In global thresholding there are many bright regions belongs to foreground and background and that are below the threshold value and therefore which results into misclassified segmented regions. Same results are shown in multilevel or color based binary image segmentation which only removes brightness of the input color image by adjusting the threshold value. Canny edge detection method gives clear results as compared to thresholding method. it detects all edges in image but extra edges are also detected which having less intensity value than intensity value on boundary. In Gradient magnitude which uses Sobel filter mask to detect edges in both directions vertical and horizontal direction and gives better results. but it is computationally more difficult because of square root and square calculations. Clustering and thresholding nearly gives same results but clustering considers all three plane of image to cluster the input image. In top-hat bottom hat dilation and erosion operations are used to extract shape of different regions but it is not accurately detect all boundary edges and some regions are not get closed. Standard deviation is used to show quantitative evaluation of results of all segmentation techniques. Standard evaluation is used to show how much output image is get deviated from the input image and with less deviation value that output image is much closer to input image. Table 2 shows results of different Segmentation Techniques based on standard deviation. From results most of the output images from gradient magnitude segmentation technique are less deviated as compare to from other segmentation technique.

\section{CONCLUSION AND FUTURE SCOPE}

In this work different image segmentation techniques are studied and analyzed and applied all segmentation techniques on food images to segment input image into different regions. From visual and quantitative evaluation gradient magnitude gives better segmentation results as compared to other segmentation technique. All segmentation techniques used for experiment are based on thresholding, edge detection and combination of edge detection methods and morphological operations. As gradient magnitude gives better results because it detect edges in vertical and horizontal direction but not taking edges in diagonal directions and computationally it is also difficult and time consuming. So consider these difficulties for more accurate and efficient results from gradient magnitude in future and for detecting overlapped regions watershed segmentation technique will be use.

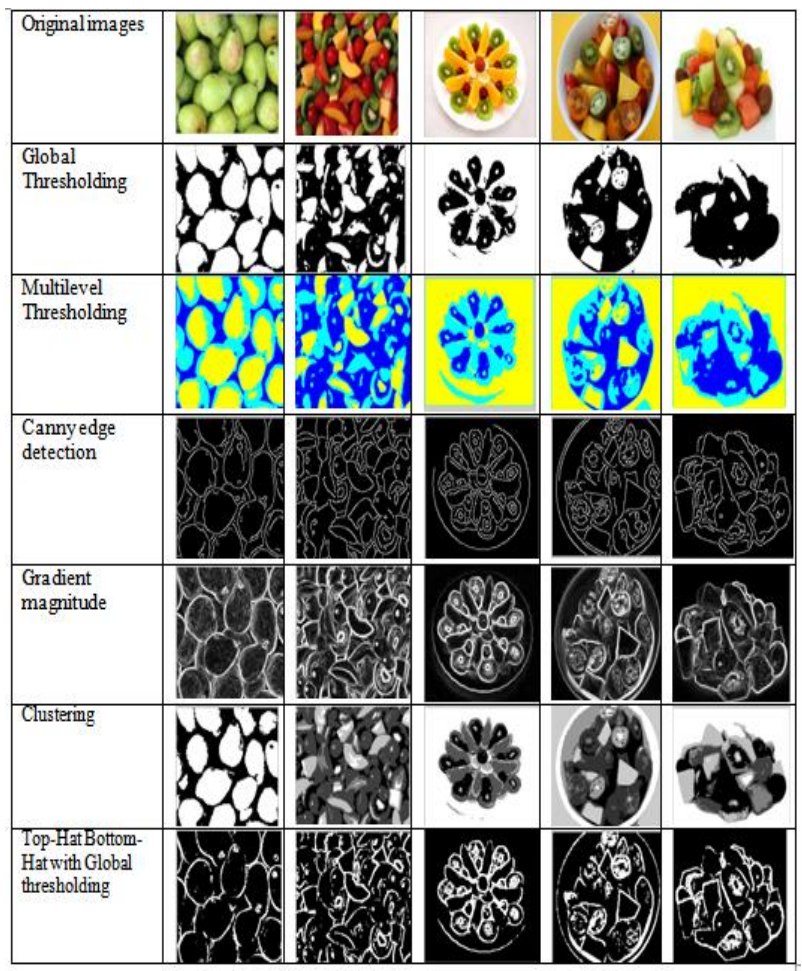

Figure 2: Output of First Five Input Images 


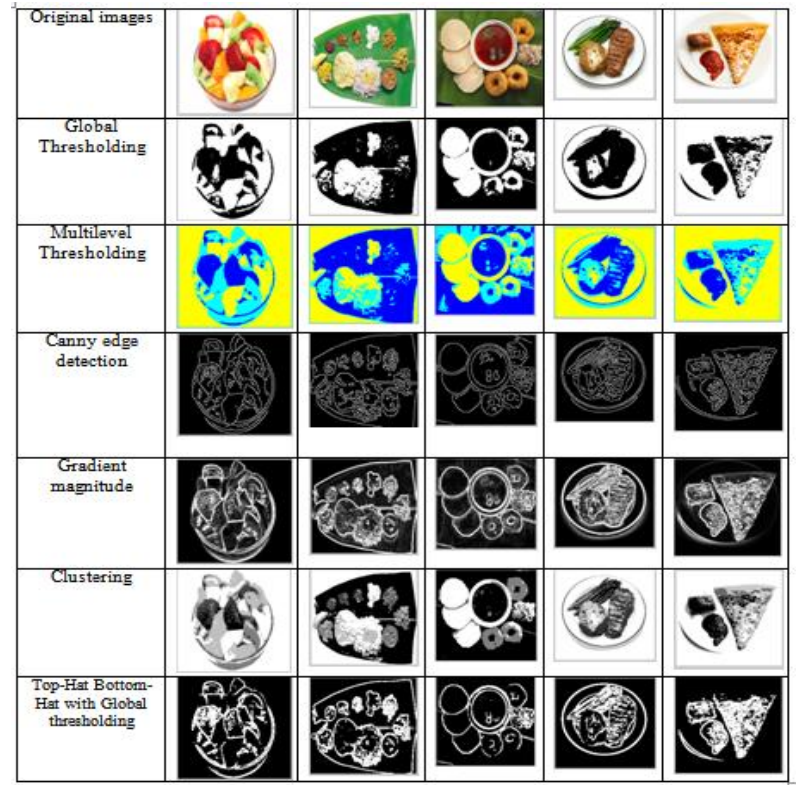

Figure 3: Output of next Five Input Images

Table 1: Comparative Analysis

\begin{tabular}{|c|c|c|c|c|}
\hline $\begin{array}{l}\text { Sr. } \\
\text { No }\end{array}$ & Paper Title & Techniques used & Input Image & Analysis \\
\hline 1 & $\begin{array}{c}\text { Measuring Calorie and } \\
\text { Nutrition From Food Image }\end{array}$ & Edge Detection & $\begin{array}{l}\text { All type of food } \\
\text { images }\end{array}$ & $\begin{array}{c}\text { Unable to segment mixed food portion or } \\
\text { overlap portions }\end{array}$ \\
\hline 2 & $\begin{array}{c}\text { Image Segmentation for } \\
\text { Food Quality Evaluation } \\
\text { Using Computer Vision } \\
\text { System }\end{array}$ & $\begin{array}{l}\text { Color based Binary Image and } \\
\text { Particle-Swarm Optimization }\end{array}$ & Food images & $\begin{array}{l}\text { It is used to find defects in food } \\
\text { materials. }\end{array}$ \\
\hline 3 & $\begin{array}{l}\text { A Rule-based Segmentation } \\
\text { Method for Fruit Images } \\
\text { Under Natural Illumination }\end{array}$ & $\begin{array}{l}\text { Otsu, K-mean, Fuzzy C-means, } \\
\text { If-Then }\end{array}$ & $\begin{array}{l}\text { Image containing } \\
\text { single food item }\end{array}$ & $\begin{array}{l}\text { It is not well suited for image containing } \\
\text { multiple food items. }\end{array}$ \\
\hline 4 & $\begin{array}{c}\text { Segmentation and } \\
\text { Recognition of Multi-Food } \\
\text { Meal Images for } \\
\text { Carbohydrate Counting }\end{array}$ & $\begin{array}{c}\text { Pyramidal Mean-shift Filtering, } \\
\text { Region Growing and Region } \\
\text { Merging }\end{array}$ & Multi food image & $\begin{array}{l}\text { Good for separate out different regions } \\
\text { having same intensity value. }\end{array}$ \\
\hline 5 & $\begin{array}{c}\text { Image Segmentation for } \\
\text { Image-Based Dietary } \\
\text { Assessment: A Comparative } \\
\text { Study }\end{array}$ & $\begin{array}{l}\text { Active Contours, Normalized } \\
\text { Cuts and Local Variation. }\end{array}$ & Multi food image & $\begin{array}{l}\text { Local Variation gives better results than } \\
\text { active contour as number of initial } \\
\text { contour has to defined in active contour. }\end{array}$ \\
\hline 6 & $\begin{array}{l}\text { Segmentation of colour food } \\
\text { images using a robust } \\
\text { algorithm }\end{array}$ & $\begin{array}{l}\text { Global Thresholding and } \\
\text { Morphological Operations. }\end{array}$ & $\begin{array}{l}\text { Image containing } \\
\text { single food item }\end{array}$ & $\begin{array}{l}\text { Good for single food item image. but in } \\
\text { multi food image it combine all regions } \\
\text { and finally gives single region. }\end{array}$ \\
\hline
\end{tabular}

Table 2: Results of Segmentation Techniques

\begin{tabular}{|c|c|c|c|c|c|c|c|}
\hline $\begin{array}{c}\text { Sr. } \\
\text { No }\end{array}$ & $\begin{array}{c}\text { Original } \\
\text { Image }\end{array}$ & Canny & $\begin{array}{c}\text { Top-Hat } \\
\text { Bottom } \\
\text { Hat }\end{array}$ & $\begin{array}{c}\text { K-means } \\
\text { clustering }\end{array}$ & $\begin{array}{c}\text { Global } \\
\text { Thresholdin } \\
\mathrm{g}\end{array}$ & $\begin{array}{c}\text { Gradient } \\
\text { Magnitude }\end{array}$ & $\begin{array}{c}\text { Colour } \\
\text { based }\end{array}$ \\
\hline 1 & 0.289 & 0.229 & 0.396 & 0.486 & 0.477 & 0.293 & 0.495 \\
\hline 2 & 0.321 & 0.239 & 0.385 & 0.356 & 0.488 & 0.311 & 0.480 \\
\hline 3 & 0.429 & 0.232 & 0.409 & 0.402 & 0.484 & 0.325 & 0.482 \\
\hline 4 & 0.384 & 0.262 & 0.409 & 0.371 & 0.486 & 0.320 & 0.484 \\
\hline 5 & 0.417 & 0.231 & 0.423 & 0.425 & 0.493 & 0.300 & 0.493 \\
\hline 6 & 0.367 & 0.254 & 0.434 & 0.379 & 0.480 & 0.395 & 0.483 \\
\hline 7 & 0.381 & 0.231 & 0.424 & 0.461 & 0.487 & 0.350 & 0.489 \\
\hline 8 & 0.310 & 0.264 & 0.407 & 0.483 & 0.487 & 0.328 & 0.490 \\
\hline 9 & 0.397 & 0.245 & 0.420 & 0.378 & 0.480 & 0.385 & 0.477 \\
\hline 10 & 0.405 & 0.238 & 0.465 & 0.428 & 0.483 & 0.359 & 0.486 \\
\hline
\end{tabular}




\section{REFERENCES}

[1] Y. He, N. Khanna, C.J. Boushey and E.J. Delp, Image Segmentation for Image-Based Dietary Assessment: A Comparative Study. 2013 IEEE.

[2] Rajeshwar Dass, Priyanka, Swapna Devi, "Image Segmentation Techniques", IJECT Vol. 3, Issue 1, Jan. March 2012

[3] Parisa Pouladzadeh, Shervin Shirmohammadi and Rana Al-Maghrabi, Measuring Calorie and Nutrition From Food Image. IEEE Transactions On Instrumentation And Measurement, Vol. 63, No. 8, August 2014.

[4] Nandhini. P and Dr. J. Jaya, Image Segmentation for Food Quality Evaluation Using Computer Vision System. Int. Journal of Engineering Research and Applications www.ijera.com ISSN : 2248-9622, Vol. 4, Issue 2( Version 5), February 2014.

[5] Hamirul Aini Hambali, Sharifah Lailee Syed Abdullah, Nursuriati Jamil and Hazaruddin Harun, A Rule-based
Segmentation Method for Fruit Images under Natural Illumination. International Conference on Computer, Control, Informatics and Its Applications 2014.

[6] Marios Anthimopoulos, Joachim Dehais, Peter Diem and Stavroula Mougiakakou, Segmentation and Recognition of Multi-Food Meal Images for Carbohydrate Counting. 2013 IEEE.

[7] Domingo Mery and Franco Pedreschi, Segmentation of colour food images using a robust algorithm. Journal of Food Engineering. 2004 Elsevier.

[8] Rozy Kumari and Narinder Sharma, A Study on the Different Image Segmentation Technique. International Journal of Engineering and Innovative Technology (IJEIT) Volume 4, Issue 1, July 2014

[9] V. K. Dehariya, S. K. Shrivastava, R. C. Jain, Clustering of Image Data Set Using K-Means and Fuzzy K-Means Algorithms, International conference on CICN, pp. 386$391,2010$. 